University of Wollongong

Research Online

Faculty of Informatics - Papers (Archive)

Faculty of Engineering and Information

Sciences

$1-5-2007$

\title{
Multi-channel digital fringe calibration for structured light profilometers using neural networks
}

\author{
Matthew J. Baker \\ matthewb@uow.edu.au \\ Jiangtao Xi \\ University of Wollongong, jiangtao@uow.edu.au \\ Joe F. Chicharo \\ University of Wollongong, chicharo@uow.edu.au
}

Follow this and additional works at: https://ro.uow.edu.au/infopapers

Part of the Physical Sciences and Mathematics Commons

\section{Recommended Citation}

Baker, Matthew J.; Xi, Jiangtao; and Chicharo, Joe F.: Multi-channel digital fringe calibration for structured light profilometers using neural networks 2007.

https://ro.uow.edu.au/infopapers/609

Research Online is the open access institutional repository for the University of Wollongong. For further information contact the UOW Library: research-pubs@uow.edu.au 


\title{
Multi-channel digital fringe calibration for structured light profilometers using neural networks
}

\author{
Abstract \\ The performance of structured light profilometers is significantly hindered by the generation of distorted \\ sinusoid fringe images, particularly, for multi-channel applications. In this paper we investigate the \\ application of neural network fringe calibration for the multi-channel approach. We analytically review the \\ nature of the major error sources associated with the multi-channel approach and propose afringe \\ calibration technique with emphasis on minimal photometric calibration. The performance of the \\ calibration technique is gauged through both simulation and experimentation.

\section{Disciplines} \\ Physical Sciences and Mathematics

\section{Publication Details} \\ This article was originally published as Baker, MJ, Xi, J, and Chicharo, J, Multi-channel Digital Fringe \\ Calibration for Structured Light Profilometers using Neural Networks, 2007 IEEE Instrumentation and \\ Measurement Technology Conference Proceedings, 1-3 May, 1-6.
}




\title{
Multi-channel Digital Fringe Calibration for Structured Light Profilometers using Neural Networks
}

\author{
M. J. Baker ${ }^{1}$, J. Xi ${ }^{1}$ and J. F. Chicharo ${ }^{1}$ \\ ${ }^{1}$ School of Electrical, Computer and Telecommunications Engineering, \\ University of Wollongong, NSW 2522, Australia \\ Tel: +6124221 3244, Fax: +61242273277 \\ E-mail: mjb06@uow.edu.au
}

\begin{abstract}
The performance of structured light profilometers is significantly hindered by the generation of distorted sinusoid fringe images, particularly, for multi-channel applications. In this paper we investigate the application of neural network fringe calibration for the multi-channel approach. We analytically review the nature of the major error sources associated with the multi-channel approach and propose a fringe calibration technique with emphasis on minimal photometric calibration. The performance of the calibration technique is gauged through both simulation and experimentation.
\end{abstract}

Keywords - Digital Video Projector, Fringe, Multi-channel, Colour, Profilometry.

\section{INTRODUCTION}

Structured light profiling has proven to be a popular alternative for fast, high precision and automated optical noncontact three-dimensional measurement in recent years. In such profilometry approaches a structured light pattern, typically, sinusoidal in nature, is projected onto a diffuse surface to be measured and viewed from an offset angle, as depicted in Figure 1. The observed fringe pattern is modulated by the object, with the modulation directly related to the depth distribution of the diffuse surface perpendicular to the plane of observation. The modulated intensity distribution is recorded, commonly by a CCD camera and processed using a fringe profilometry technique such as the popular Phase Measuring (PMP) [1] or Fourier Transform (FTP) [2] Profilometry methods, to obtain the spatial modulation and hence spatial displacement $A C$. Exploiting the geometrical relationship, $\triangle E_{p} E_{c} D \sim \triangle A C D$, apparent in the physical arrangement the spatial modulation is thereby used to recreate the surface $h(x, y)$ in three-dimensional space, generally with a high degree of precision.

$$
h(x, y)=\frac{l_{0} A C}{A C-d_{0}},
$$

Traditionally, structured light fringe patterns have been generated using interferometric methods via a laser source. An alternative to conventional laser projection is Digital Video Projection (DVP). DVP is a technology which has been actively

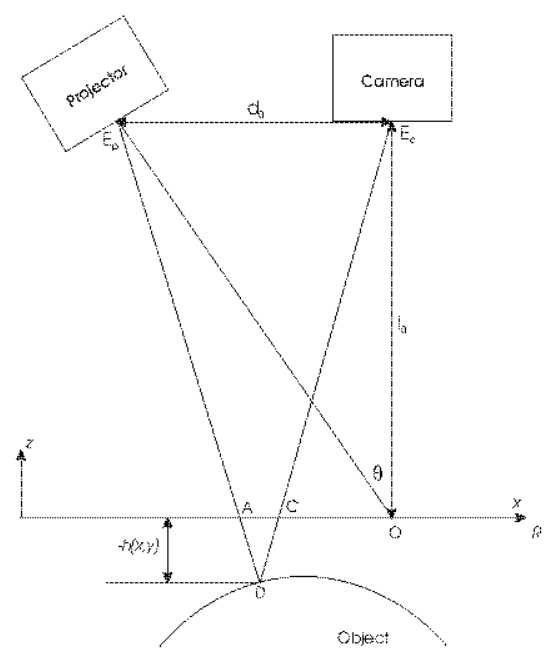

Fig. 1. Typical Crossed Optical Axes profilometry arrangement

pursued in this particular field of research as it can provide a number of key advantages. For instance, DVP provides the ability to manipulate fringe patterns quickly and easily with high precision in software, and hence when interfaced to a personal computer makes a very affordable, flexible and robust projection source. Furthermore, the application of DVP colour projection functionality, provides the means to increase fringe acquisition speed by a factor of three, hence, resulting in the more recent development of dynamic multichannel profiling algorithms. Advancements in the performance of such digital technology in conjunction with these incentives have fueled continued interest from the research community over recent years. However, for techniques such as PMP and FTP, which are nonresilient to fringe anomaly such as gamma non-linear luminance effects [3] and colour channel coupling (in the multichannel case) commonplace with DVP, system accuracy is significantly hindered.

In the past solutions to counter such fringe anomalies typically involve photometric calibration. To alleviate the non- 
linear luminance effects multiple intensity distributions varying over the full range of luminance values are recorded and a camera / projector luminance curve is fitted [4], [5]. Similarly to facilitate the adequate separation of a multi-channel fringe into its individual colour components, a number of colour images are projected / captured and a mixing matrix representing the portion of colour leakage to and from each channel is formed[4], [6]. Often such approaches can be laborious, time consuming, require a substantial amount of additional data and are typically nonresilent to environmental change.

Recently, the successful application of neural networks to remove fringe non-linearities was presented [7]. The approach exploits the generalisation properties of a feed-forward backpropagation neural network predicting the inverse non-linear fringe transform function, consequentially, improving the determination of spatial displacement $A C$ and therefore $h(x, y)$. Such an approach is attractive as no additional data is required for calibration. Hence, the fringe calibration technique lends itself as an ideal solution for multi-channel fringe calibration bearing in mind the considerable amount of data required for photometric calibration.

In this paper we investigate the application of neural networks for DVP multi-channel fringe calibration. The remainder of this paper is set out as follows; Firstly, we describe the principle multi-channel PMP approach and provide analytical investigation into both Gamma luminance and Colour channel coupling fringe anomalies and the implication they both bear on the phase measuring approach. Section IV outlines the proposed calibration technique with emphasis on minimal photometric calibration, with Sections V and VI providing simulation and empirical validation of the proposed approach.

\section{PRINCIPLE DIGITAL MULTI-CHANNEL FRINGE PROFILOMETRY}

The ability to project and acquire colour fringe images has made possible the realtime application of popular non-single shot approaches such as PMP. Probably the most exploited multi-channel technique is the 3 Step PMP approach since all required data can be obtained in a single exposure. Given that PMP processes $A C$ as a spatial phase displacement, requiring the projection of sinusoidal intensity distributions with spatial frequency $f_{0}$, Equation (1) becomes

$$
h(x, y) \approx-\frac{l_{0}}{2 \pi f_{0} d_{0}} \Delta \phi(x, y),
$$

where

$$
\Delta \phi(x, y)=\phi(x, y)-\phi_{0}(x, y)
$$

and where $\phi(x, y)$ and $\phi_{0}(x, y)$ are the phase modulations relating to the diffuse surface to be profiled and the reference plane $R$ respectively, for the case where $l_{0} \gg h(x)$.
For the PMP case where $N$ phase shifted images are required we can obtain spatial phase modulation $\Delta \phi(x, y)$ as

$$
\begin{aligned}
\Delta \phi(x, y)= & -\tan ^{-1}\left[\frac{\sum_{n=0}^{N-1} g_{n}(x, y) \sin (2 \pi n / N)}{\sum_{n=0}^{N-1} g_{n}(x, y) \cos (2 \pi n / N)}\right] \\
& +\tan ^{-1}\left[\frac{\sum_{n=0}^{N-1} g_{0 n}(x, y) \sin (2 \pi n / N)}{\sum_{n=0}^{N-1} g_{0 n}(x, y) \cos (2 \pi n / N)}\right]
\end{aligned}
$$

where the captured fringes are

$$
\begin{array}{r}
g_{n}(x, y)=a(x, y)+b(x, y) \cos \left(2 \pi f_{0} x+\phi(x, y)\right. \\
+2 \pi n / N) \\
\text { and } \\
g_{0 n}(x, y)=a(x, y)+b(x, y) \cos \left(2 \pi f_{0} x+\phi_{0}(x, y)\right. \\
+2 \pi n / N) \\
\text { for } \mathrm{n}=0,1,2, \ldots, \mathrm{N}-1,
\end{array}
$$

where $a(x, y)$ and $b(x, y)$ are functions representing the captured fringe offset and contrast.

Observing Equation (4), it is quite clear that the mathematics governing the evaluation of spatial displacement $A C$ and hence $h(x, y)$, requires the adequate generation and capture of pure sinusoidal fringe images. In practice for DVP based approaches it quickly becomes apparent that the signals acquired at the respective CCD's of the capture device are clearly not pure sinusoid signals and hence the accuracy of the PMP multichannel reconstruction is inadvertently compromised. Typical DVP multi-channel fringes often present non-linear luminance anomalies and further fringe distortion resulting from the coupling of colour channels which need to be compensated for prior to use with phase measuring approaches. We now investigate the nature and implications such fringe anomalies pose for phase measuring profilometry approaches.

\section{DIGITAL MULTI-CHANNEL FRINGE ANOMALY A. Gamma Distortion}

Gamma distortion is typical in visual display systems to enhance human perception of the sensation of lightness, which can be regarded as a power function of intensity [8]. The $\gamma$ of a DVP device is an inherent and individual feature, specific to each colour channel, which is typically optimised to enhance human visual perception of projected images for applications such as home theater and video presentation. In general, the gamma distortion of a digital display can be modeled using the simple power function seen in Equation (6)

$$
\bar{u}(x, y)=u(x, y)^{\gamma}, \quad \text { for } u \in[0,1]
$$

where $u(x, y)$ is the normalised image delivered to the display device, $\bar{u}(x, y)$ is the actual normalised image output intensity distribution and $\gamma$ is typically a fractional value $>1$ specific to the display device. For the projection of a sinusoid this results 
in the harmonics and thus we can represent a projected fringe as a Fourier Series with infinite $m$ th order harmonic components. Considering only the contribution of the 2 nd order harmonic we can define

$$
p=\frac{\left[a^{\gamma} \int_{0}^{T}\left[1+\frac{b}{a} \cos \left(2 \pi f_{0} x\right)\right]^{\gamma} \cos \left(4 \pi f_{0} x\right) d x\right]^{2}}{\left[a^{\gamma} \int_{0}^{T}\left[1+\frac{b}{a} \cos \left(2 \pi f_{0} x\right)\right]^{\gamma} \cos \left(2 \pi f_{0} x\right) d x\right]^{2}}
$$

the power ratio of the 2 nd order harmonic to the fundamental, which is a function of the ratio of fringe contrast and offset parameters $\frac{b}{a}$ and $\gamma$, where $T$ corresponds to the spatial period of the fringe image.

For the multi-channel case $\gamma$ varies for each colour channel, however, for simplicity to gain an understand of the implications of $\gamma$ we consider just the single channel application where $\gamma_{R}=\gamma_{G}=\gamma_{B}$. We now denote $\delta$ as the phase measuring error associated with the 2 nd order sensitive multi-channel case and also let $\theta$ denote $2 \pi f_{0} x+\phi(x, y)$, and show that

$$
\delta=-\tan ^{-1}\left(\frac{\sqrt{p} \sin (3 \theta)}{1+\sqrt{p} \cos (3 \theta)}\right)
$$

representing the phase error function associated with 2 nd order fringe harmonic distortion. And it can be further shown that the maximum absolute phase measuring error can be given as

$$
\varepsilon_{\max }=2 \cdot \tan ^{-1}\left(\sqrt{\frac{p}{1-p}}\right)
$$

and hence using Equation (2), the maximum absolute height measurement error $\beta$ is

$$
\beta=\frac{l_{0}}{\pi f_{0} d_{0}} \cdot \tan ^{-1}\left(\sqrt{\frac{p}{1-p}}\right)
$$

[9]. If we now assume system parameters $l_{0}, d_{0}$ and $f_{0}$ are $5 \mathrm{~m}, 2 \mathrm{~m}$ and $10 / \mathrm{m}$ respectively, we can plot a curve describing the relationship, as depicted in Figure (2). It can be seen that for larger values of $\gamma$ i.e. stronger harmonics, the greater the profile measurement error.

\section{B. Coupling of Colour Channels}

The generation of colour in any digital projection display device involves the use of dichroism. Typically, dichroic filters or prisms are used to separate a white light source into it's primary components, by transmitting or reflecting the appropriate wavelengths of light, and therefore, producing the sensation referred to as colour. Since coloured light can be regarded as a spectral distribution where power is a function of wavelength, through the trichromacy and metamerism principles [10] a projection device can produce controlled colour by adding weighted spectral distributions obtained from the

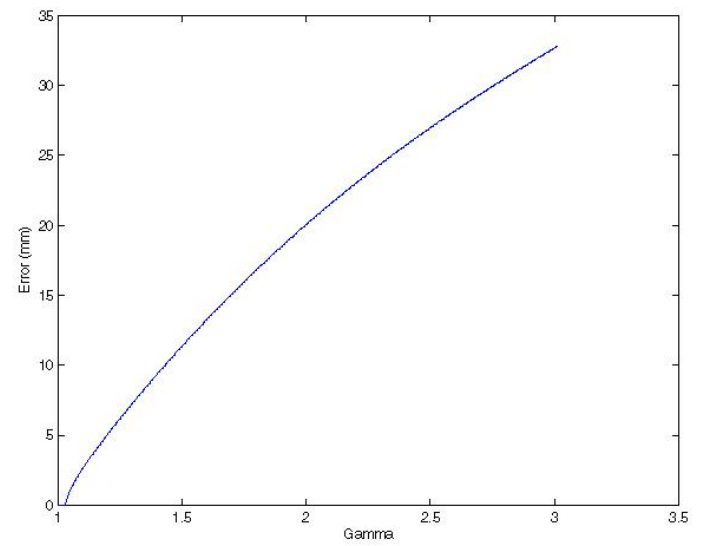

Fig. 2. Maximum absolute profile measurement error: for fringe parameters $a=b$

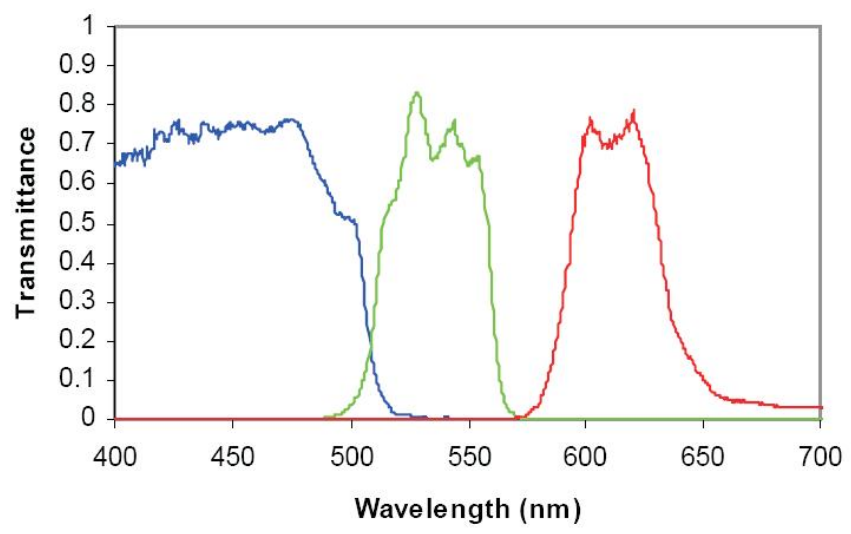

Fig. 3. CCD Filter response

dichroic separation of the white light source within the projectior. Mathematically, to represent a colour with power spectral distribution $c(\lambda)$,

$$
c(\lambda)=R \cdot \overline{c_{r}}(\lambda)+G \cdot \overline{c_{g}}(\lambda)+B \cdot \overline{c_{b}}(\lambda)
$$

where $\overline{c_{r}}(\lambda), \overline{c_{g}}(\lambda)$ and $\overline{c_{b}}(\lambda)$ are the primary spectra, with corresponding RGB tristimulus values. Therefore, from the software perspective an appropriate colour model, namely RGB, is required to link the tristimulus values and the desired colour response. The spectral distribution of each of the defined primaries $\overline{c_{r}}(\lambda), \overline{c_{g}}(\lambda)$ and $\overline{c_{b}}(\lambda)$ for all $\lambda$ are typically non-zero over common intervals, in other words, overlaps in the spectra exists for each of the primary sources. As a result colour leaks to and from each of the primaries, the phenomenon is often referred to as coupling of colour channels. Furthermore, the dichroic optics responsible for the representation of colour within the capture device also incurs additional overlapping and further heightens the colour channel coupling. The typical filter response for a 3-CCD camera is shown in Figure 3.

For the projection / acquisition of multi-channel fringe im- 
ages for phase measuring approaches where accurate isolation of phase shifted sequential fringes is critical, the coupling of colour channels significantly impacts performance. Particularly, when combined with the individual channel gamma nonlinearities, many fringe deformations are introduced. However, since the spectra overlap for both the acquisition and projection stages is constant, the leakage is predictable and can be modeled as

$$
\left(\begin{array}{c}
r(\hat{x}, y) \\
g(\hat{x}, y) \\
b(\hat{x}, y)
\end{array}\right)=\left(\begin{array}{ccc}
1 & k_{G R} & k_{B R} \\
k_{R G} & 1 & k_{B G} \\
k_{R B} & k_{G B} & 1
\end{array}\right) \cdot\left(\begin{array}{c}
r(x, y) \\
g(x, y) \\
b(x, y)
\end{array}\right)(12)
$$

where $r(\hat{x}, y), g(\hat{x}, y)$ and $b(\hat{x}, y)$ represent the signals obtained at the respective colour CCD sensors of the acquisition device, $k_{X Y}$ represents the normalised proportion of colour coupling between the appropriate channels and $r(x, y), g(x, y)$ and $b(x, y)$ correspond to the actual intended spectral distributions prior to projection.

To investigate the impact the coupling of colour channels has on the phase measuring algorithm we assume similar linear channel luminance $\gamma_{R}=\gamma_{G}=\gamma_{B}=1$. Clearly this is not true in practice, however, such assumptions help provide insight into the nature and also gauge the magnitude of resulting phase measuring error. We can thereby denote the general amplitude, $\psi_{n}$ and phase angle, $\zeta_{n}$, of a crosstalk fringe as

$$
\begin{gathered}
\psi_{n}=\sqrt{3\left(k_{n 1}-k_{n 2}\right)^{2}+\left(2 k_{n 0}-k_{n 1}-k_{n 2}\right)^{2}} \\
\zeta_{n}=\tan ^{-1}\left(\frac{\sqrt{3}\left(k_{n 1}-k_{n 2}\right)}{2 k_{n 0}-k_{n 1}-k_{n 2}}\right)
\end{gathered}
$$

where $k_{n 0}, k_{n 1}$ and $k_{n 2}$ for $0 \leq k_{n, 012}<1$ denote the normalised proportion of channels 1,2 and 3 , respectively, for the $n$th fringe image with corresponding $0,2 \pi / 3$ and $4 \pi / 3$ phase shift. If we now assume the amplitude components of each fringe have been normalised i.e. $\psi_{n}=\psi_{n}=\psi_{n}=\psi$, we can see that a phase error for each fringe will be propagated through the phase measuring algorithm

$$
\hat{\theta}=\tan ^{-1}\left(\frac{\sqrt{3}\left(\cos \left(\theta+\zeta_{1}\right)-\cos \left(\theta+\zeta_{2}\right)\right)}{2 \cos \left(\theta+\zeta_{0}\right)-\cos \left(\theta+\zeta_{1}\right)-\cos \left(\theta+\zeta_{2}\right)}\right)
$$

where $\hat{\theta}$ represents the predicted value of $\theta$, as evaluated in the presence of the colour coupling. Substituting Equation (14) into Equation (15) it can be shown that the erroneous phase prediction $\hat{\theta}$ can be given as

$$
\hat{\theta}=\tan ^{-1}\left(\frac{\sqrt{3}(\alpha \cos \theta+\sqrt{3} \eta \sin \theta)}{\rho \cos \theta+\sqrt{3} \chi \sin \theta}\right)
$$

where

$$
\alpha=2\left(k_{10}-k_{20}\right)-k_{12}+k_{21}
$$

$$
\begin{aligned}
& \eta=k_{21}+k_{12}-2 \\
& \rho=6-2\left(k_{01}+k_{02}+k_{10}+k_{20}\right)+k_{12}+k_{21} \\
& \chi=2\left(k_{02}-k_{01}\right)-k_{12}+k_{21}
\end{aligned}
$$

Hence, it can be seen that the error introduced by the coupling of the colour channels is a function of the four crosstalk variables, $\alpha, \beta, \epsilon$ and $\chi$ and also $\theta$, and thus we can conclude the error will not only depend on the amount of crosstalk but also on the surface to be profiled.

\section{PROPOSED FRINGE CALIBRATION TECHNIQUE}

Due to the nature of the multi-channel fringe anomalies we propose a two step calibration process; firstly the colour channel coupling is removed, followed by the intensity linearisation / normalisation of each channel.

\section{A. Counter Coupling of Colour Channels}

For most commercial digital video projectors, significant leakage from just one channel is typically observed, and therefore, only two coupling factors are considered influential. Now considering just two coupling factors and examining Equation (16) it can be revealed that colour channel selection would not likely provide for significant improvement in the prediction of $\theta$. Hence, in order to ensure the accurate prediction of $\theta$ the accurate prediction of mixing matrix $K$ and thus isolation of each fringe is essential. If $K$ is known the coupling can be removed as follows

$$
\left(\begin{array}{l}
r(x, y) \\
g(x, y) \\
b(x, y)
\end{array}\right)=\hat{K} \cdot\left(\begin{array}{c}
r(\hat{x}, y) \\
g(\hat{x}, y) \\
b(\hat{x}, y)
\end{array}\right)
$$

where

$$
\hat{K}=\left(\begin{array}{ccc}
1 & -k_{G R} & -k_{B R} \\
-k_{R G} & 1 & -k_{B G} \\
-k_{R B} & -k_{G B} & 1
\end{array}\right)
$$

Since $\hat{K}$ is a distinctive variable set for each projector camera arrangement it must be measured by photometric processes.

\section{B. Gamma Non-linear Luminance}

Subsequent to the counter coupling of colour channels the intensity of each channel is linearised and normalised via a neural network based approach. The neural network based technique endeavors to produce a pure sinusoid fringe from a gamma distorted fringe via a multi-layer feedforward backpropagation neural network operating as a nonlinear signal map. The technique considers $\overline{r_{n}}(x)$, a single cross-section of a digitised gamma distorted multi-channel fringe image projected onto a reference plane (where $n$ corresponds to the appropriate channel). The gamma non-linearity is assumed constant over the entirety of the captured image for each channel and a training vector $t_{n}(x)$ is formed for each channel as

$$
t_{n}(x)=a+b \cos \left(2 \pi f_{0} x+\phi_{0}(x)+2 \pi n / 3\right),
$$




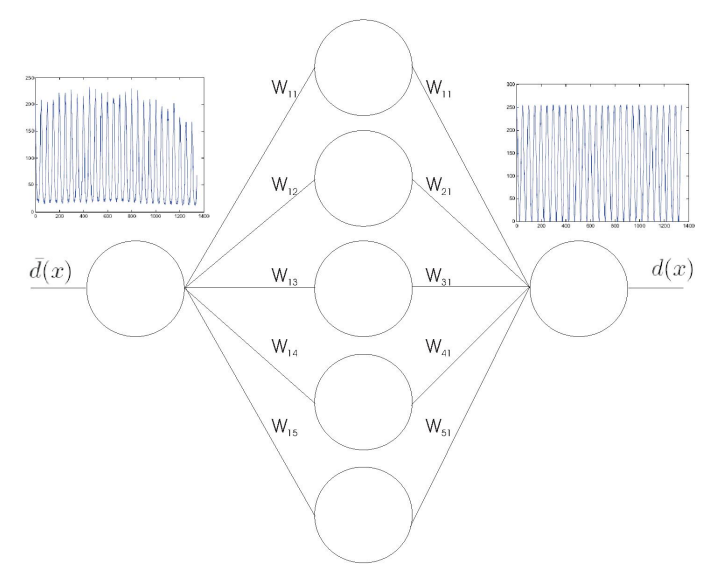

Fig. 4. Proposed Multilayer Signal Mapping Calibration Neural Network

The training vectors represents the anticipated or desired fringe intensity distribution, such as the software input to each colour channel of the digital projection source with included phase modulation and initial phase. Three neural networks such as that depicted in Figure (4) are trained to map sample by sample from the distorted vectors, $\overline{r_{n}}(x)$, to the target vectors.

Since the $\gamma$ of the display in each channel is assumed constant using the trained neural networks a calibrated reference fringe image, $r_{n}(x, y)$, can be produced as follows

$$
\begin{array}{r}
r_{n}(x, y)=f_{2}\left(\sum W_{n_{2}} f_{1}\left(\overline{r_{n}}(x, y) W_{n_{1}}\right)\right) \\
\text { for } \forall x, \\
\text { and } \forall y,
\end{array}
$$

where $W_{n_{1}}$ and $W_{n_{2}}$ and $f_{1}$ and $f_{2}$ are the appropriate weight matrices and activation functions for the corresponding layers and respective channels.

Once the reference plane fringe sequence has been calibrated the same neural networks are used to calibrate the deformed fringes, $\bar{d}_{n}(x, y)$, also

$$
\begin{array}{r}
d_{n}(x, y)=f_{2}\left(\sum W_{n_{2}} f_{1}\left(\bar{d}_{n}(x, y) W_{n_{1}}\right)\right) \\
\text { for } \forall x, \\
\text { and } \forall y,
\end{array}
$$

The fringe sequences $r_{n}(x, y)$ and $d_{n}(x, y)$ are then used by the phase measuring algorithm to predict $\theta$.

\section{SIMULATION}

To validate and also gauge the performance of the proposed calibration technique we simulate the reconstruction of a diffuse surface $h(x, y)$, illuminated by the multi-channel fringe described as follows

$$
\left(\begin{array}{c}
r(\hat{x, y}) \\
g(\hat{x, y}) \\
b(\hat{x, y})
\end{array}\right)=K\left(\begin{array}{c}
{\left[a+b \cos \left(2 \pi f_{0} x\right)\right]^{\gamma_{R}}} \\
{\left[a+b \cos \left(2 \pi f_{0} x+2 \pi / 3\right)\right]^{\gamma_{G}}} \\
{\left[a+b \cos \left(2 \pi f_{0} x+4 \pi / 3\right)\right]^{\gamma_{B}}}
\end{array}\right)
$$

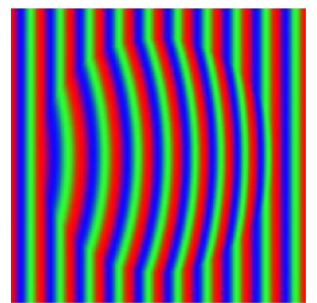

(a) Typical Multi-channel Fringe

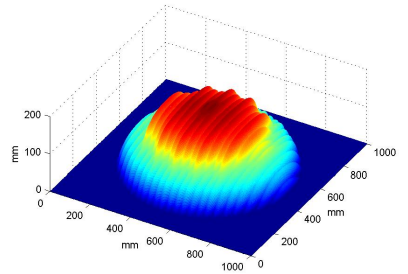

(c) Non-calibrated reconstruction

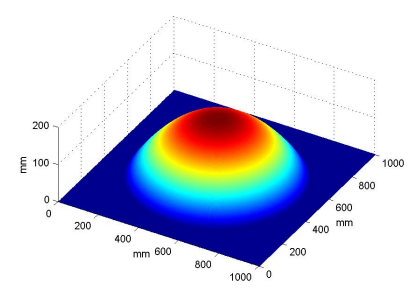

(b) Simulated 3D surface, $h(x, y)$
Fig. 5. Simulated reconstructed surface with and without the proposed fringe calibration

\begin{tabular}{l|cc} 
& $\operatorname{PMP}(N=3)$ & \\
\hline & $\epsilon(\mathrm{mm})$ & $\delta(\mathrm{mm})$ \\
\hline Non-Calibrated & 5.443 & 5.4967 \\
Calibrated & 0.0403 & 0.0427
\end{tabular}

TABLE I. Calibrated and Non-calibrated mean absolute reconstruction errors and standard deviations.

$\gamma_{r}, \gamma_{g}$ and $\gamma_{b}$ were chosen to be $2.2,2.5$, and 2.1 respectively with an appropriately simulated mixing matrix $K$. System parameters $l_{0}, d_{0}$ and $f_{0}$ were chosen to be $5 \mathrm{~m}$ and $2 \mathrm{~m}$ and $10 / \mathrm{m}$ respectively, corresponding to a spatial period of $100 \mathrm{~mm}$ if we assume an image spatial resolution of 1pixel/mm. Fringe parameters $a$ and $b$ were chosen to be equivalent to maximise the influence of the non-linear $\gamma$ distortion. We reconstruct the non-calibrated and calibrated fringe images using the phase measuring algorithm. Table I displays the mean absolute measuring error $\epsilon$ and standard deviation $\delta$ for both the calibrated and non-calibrated cases. A visual representation of the simulated fringe, hemispherical test surface and calibrated and non-calibrated reconstructions can be seen in Figure 5. The improvement for the calibrated fringe in contrast to the noncalibrated is clearly evident visually.

\section{EXPERIMENTAL}

In order to verify the physical application of the multichannel fringe calibration approach, practical experimental results were established through the profiling of a diffuse surface. A multi-channel sinusoidal fringe pattern was projected using a Hitachi CP-X260 LCD digital video projector and cap- 


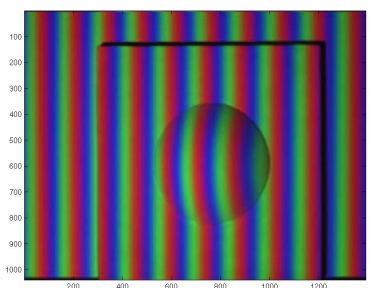

(a) Experimental surface

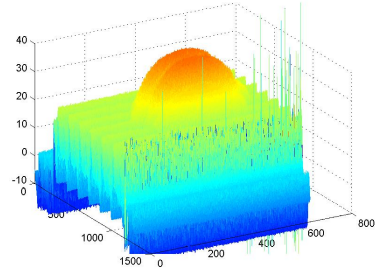

(c) Non-calibrated Reconstruction

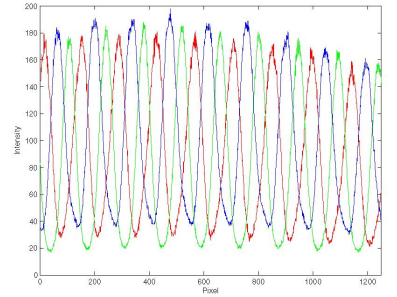

(b) Reference Fringe Crosssection

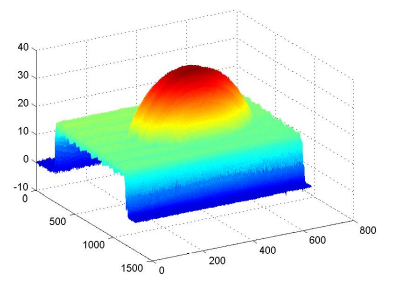

(d) Calibrated Reconstruction
Fig. 6. Experimental Results

tured using a DuncanTech MS3100 3-CCD camera. The spatial resolution of the captured fringe image was measured to be $0.215 \mathrm{~mm} /$ pixel, yielding a spatial period of $30.9 \mathrm{~mm}$ equating to a spatial frequency $f_{0}$ of 32.4 fringes $/ \mathrm{m}$. System parameters $l_{0}$ and $d_{0}$ were measured to be approximately $1.405 \mathrm{~m}$ and $0.465 \mathrm{~m}$ respectively. The profiled surface was a convex dome shape as seen in Figure 6(a). The maximum height of the hemispherical surface is $22.8 \mathrm{~mm}$ with a diameter of $99 \mathrm{~mm}$ with the thickness of the base material being $16 \mathrm{~mm}$.

It was found that the only significant colour channel coupling was from the green to red and green to blue channels, all other terms in the mixing matrix were ignored. The photometric measurement of $k_{r g} k_{b g}$ was undertaken via the projection of a pure green fringe and the red and blue components were measured. To ensure the integrity of the assumption of a static mixing matrix a high intensity fringe was projected, with $a=150$ and $b=100$. Despite our efforts to justify a uniform colour mixing, the colour mixing presented spatial attributes.

The projected fringe was filtered using a gaussian smoothing technique to maximise the performance of the neural approach. The three neural networks as described in Section IV were used to counter the channel $\gamma$ non-linearity sample by sample. The reconstructed surfaces for the non-calibrated and calibrated fringes is shown as Figures 6 (c) and (d) respectively. A cross-section of the reconstructed surface for both the calibrated and non-calibrated cases is shown in Figures 7 (a) and (b). The calibration technique has clearly been successful in minimising the associated fringe errors, however, a significant phase measuring error is still evident as indicated in Figure 7. The error can be attributed to the inadequate isolation of each colour channel, which then led to the poor training of the neural networks. It is believed that the spatial aspects of the colour

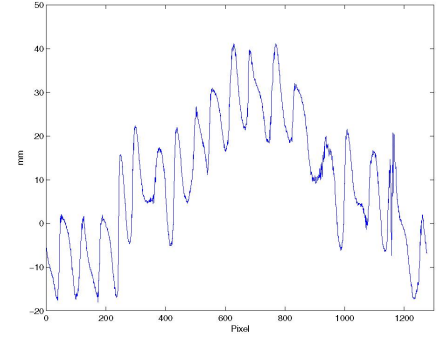

(a) Non-calibrated Reconstruction

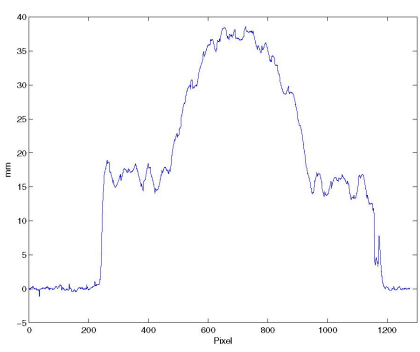

(b) Calibrated Reconstruction
Fig. 7. Cross-section of reconstructed diffuse surface seen in Figure 6(a)

coupling is the predominant phenomena inhibiting the performance of the proposed approach.

\section{CONCLUSION}

In this paper we have investigated the multi-channel application of neural network based fringe calibration for structured light profilometers. Analytical investigation into the major sources of error has been provided and consequently we proposed a multi-channel fringe calibration approach with minimal photometric calibration. The proposed technique has been verified through simulation, and, preliminary experimental results also reveal significantly improved surface profile reconstruction. However, from our empirical study the isolation of each colour channel still poses as a major issue for such approaches resulting in significant phase measuring error. In future work we would like to completely eliminate the photometric calibration from our approach and hence further research into the appropriate elimination of the coupling of colour channels will be undertaken.

[1] V. Srinivasan, H. C. Lui, M. Halioua, Automated phase-measuring profilometry of 3-d diffuse objects, Applied Optics 23 (1984) 3105-3108.

[2] X. Su, W. Chen, Fourier transform profilometry: a review, Optics and Lasers in Engineering 35 (2001) 263-284.

[3] H. Guo, H. He, M. Chen, Gamma correction for digital fringe projection profilometry, Applied Optics 43 (14) (2004) 2906 - 2914.

[4] P. S. Huang, Q. Hu, F. Jin, F. Chiang, "Color-encoded digital fringe projection technique for high speed three-dimensional surface contouring," Optical Engineering 38, pp. 1065-1071, 1999.

[5] S. Zhang, P. Huang, "High-resolution, real-time 3d shape acquisition," in 2004 Conference on Computer Vision and Pattern Recognition Workshop (CVPRN'04), (Washington D.C USA), June - July 2004.

[6] L. Kinell, Multichannel method for absolute shape measurement using projected fringes, Optics and Lasers in Engineering 41 (2004) 57-71.

[7] M. J. Baker, J. Xi, J. F. Chicharo, Neural network digital fringe calibration technique for structure light profilometers, Applied Optics 46 (8) (2007) 1233 - 1243 .

[8] C. A. Poynton, Gamma and it Disguises: The Nonlinear Mappings of Intensity in Perception, CRTs, Film and Video, SMPTE Journal, December (1993) 1099-1108.

[9] Y. Hu, J. Xi, E. Li, J. Chicharo, Z. Yang, Three-dimensional profilometry based on shift estimation of projected fringe patterns, Applied Optics 45 (4) (2006) $678-687$.

[10] M. C. Stone, Representing Colors as Three Numbers, Tutorial IEEE Computer Graphics and Applications July / August (2005) 78 - 85. 